\title{
Cypellogins A, B and C, Acylated Flavonol Glycosides from Eucalyptus cypellocarpa
}

\author{
Naoki Kasajima, ${ }^{a}$ Hideyuki Ito, ${ }^{b}$ Tsutomu Hatano, ${ }^{b}$ Takashi Yoshida, ${ }^{b}$ and Miyuki KanedA ${ }^{*}, a$ \\ ${ }^{a}$ School of Pharmacy, Shujitsu University; Nishigawara, Okayama 703-8516, Japan: and ${ }^{b}$ Faculty of Pharmaceutical \\ Sciences, Okayama University; Tsushima, Okayama 700-8530, Japan. \\ Received May 16, 2005; accepted July 14, 2005; published online July 15, 2005
}

Three new acylated flavonol glycosides, cypellogins A (1), B (2) and C (3), along with eight known phenolic compounds, were isolated from the dried leaves of Eucalyptus cypellocarpa, and their structures were elucidated using spectroscopic methods, including 2D NMR experiments and chemical evidence.

Key words Eucalyptus cypellocarpa; Myrtaceae; acylated quercetin glycoside; oleuropeic acid ester; cypellogin

Myrtaceous plants are known to be rich sources of biologically active terpenoids, tannins, flavonoids, and phloroglucinol derivatives. ${ }^{1,2}$ In the course of our studies on the polyphenolic constituents of myrtaceous plants, we reported the isolation of ten polyphenolic constituents including three new acylated phenol glucosides, cypellocarpins A, B and C, ${ }^{3)}$ from the leaf extract of Eucalyptus cypellocarpa L. A. S. (Myrtaceae), which is widely distributed in Australia and is one of the plants eaten by koalas. Further investigation into this plant has led to the isolation of 11 polyphenols, including three new acylated flavonol glycosides, cypellogins A, B and $\mathrm{C}$. This paper describes the structure of these new compounds.

A $\mathrm{MeOH}$ extract of the dried leaves of E. cypellocarpa was suspended in $\mathrm{H}_{2} \mathrm{O}$ and extracted successively with $n$ hexane, AcOEt and $n$-BuOH. The AcOEt extract was fractionated by a combination of column chromatography on Diaion HP-20, Toyopearl HW-40, YMC-GEL ODS AQ 120$50 \mathrm{~S}$ and preparative HPLC to yield cypellogins A (1), B (2) and $\mathrm{C}$ (3) and eight known compounds. The known compounds were identified as icariside $\mathrm{H}_{1}{ }^{4)}$ benzyl $6^{\prime}$ - $O$-galloyl$\beta$-glucopyranoside, ${ }^{5)}$ quercetin 3-O-(6" $O$-galloyl $)-\beta$-D-galactopyranoside, 3-O-methylellagic acid $4^{\prime}-O$-rhamnoside, ${ }^{6}$ resinoside $\mathrm{A},{ }^{7)}$ resinoside $\mathrm{B},{ }^{7)}$ guaijaverin and quercitrin, by direct comparison with authentic samples and spectroscopic comparisons with data in the literature.

Cypellogin A (1) was obtained as a light yellow amorphous powder and its molecular formula, $\mathrm{C}_{31} \mathrm{H}_{34} \mathrm{O}_{14}$, was established from a pseudomolecular ion peak at $\mathrm{m} / \mathrm{z} 653.1852$ $[\mathrm{M}+\mathrm{Na}]^{+}$in a high resolution electrospray ionization mass spectrum (HR-ESI-MS). The presence of isoquercitrin moiety in the molecule was indicated by UV ( $\lambda_{\max } 204,257$, $356 \mathrm{~nm}$ ) and ${ }^{1} \mathrm{H}-\mathrm{NMR}$ spectra which disclosed signals due to a hydrogen-bonded $\mathrm{OH}$ group $[\delta 12.47(1 \mathrm{H}, \mathrm{s})]$, an $\mathrm{ABX}$ system $[\delta 7.74(1 \mathrm{H}, \mathrm{d}, J=1.5 \mathrm{~Hz}), 7.66(1 \mathrm{H}, \mathrm{dd}, J=1.5$,
$8.0 \mathrm{~Hz}), 6.90(1 \mathrm{H}, \mathrm{d}, J=8.0 \mathrm{~Hz})]$ and two aromatic protons $[\delta$ $6.48(1 \mathrm{H}, \mathrm{d}, J=2.0 \mathrm{~Hz}), 6.25(1 \mathrm{H}, \mathrm{d}, J=2.0 \mathrm{~Hz})]$ as well as those characteristic of $\beta$-D-glucopyranose. This partial structure was also substantiated by the ${ }^{13} \mathrm{C}$-NMR spectrum of $\mathbf{1}$, in which 21 signals in total 31 carbon signals were assignable to quercetin and glucose moieties. The remaining 10 carbon signals composed of seven $s p^{3}(\delta 72.0,44.9,28.0,27.3,26.8$, $25.7,23.9)$ and three $s p^{2}$ carbon signals ( $\delta$ 167.2, 140.5, $130.5)$, along with ${ }^{1} \mathrm{H}-\mathrm{NMR}$ signals due to an olefinic [ $\delta$ $6.72(1 \mathrm{H}, \mathrm{m})]$, two tertiary methyls $[\delta 1.15,1.14$ (each $3 \mathrm{H}$, each s)], a methine $[\delta 1.40(1 \mathrm{H}, \mathrm{m})]$ and three methylene protons $[\delta 1.0-2.3]$, indicated the presence of an oleuropeic acid unit found in the co-existing cypellocarpins $\mathrm{A}-\mathrm{C}$ in the same plant. ${ }^{3)}$ The oleuropeic acid moiety was confirmed by the methanolysis of $\mathbf{1}$ with $\mathrm{NaOMe}$ in $\mathrm{MeOH}$, which gave the $(+)$-oleuropeic acid methyl ester (4) $\left\{[\alpha]_{\mathrm{D}}^{20}+85^{\circ}\right.$ $\left.\left(\mathrm{CHCl}_{3}\right)\right\} .^{3,8)}$ The allocation of the glucose and oleuropeic acid residues was confirmed by significant three-bond correlations between $\delta 134.9(\mathrm{C}-3)$ and $\delta 5.47\left(\mathrm{H}-1^{\prime \prime}\right)$, and between $\delta 167.2\left(\mathrm{C}-7^{\prime \prime \prime}\right)$ and $\delta 6.72\left(\mathrm{H}-2^{\prime \prime \prime}\right), 4.34\left(\mathrm{H}-6^{\prime \prime}\right)$ and $4.08\left(\mathrm{H}-6^{\prime \prime}\right)$ in the ${ }^{1} \mathrm{H}$-detected heteronuclear multiple bond connectivity (HMBC) spectrum. Glucose liberated on the acid hydrolysis of $\mathbf{1}$ was evidenced as D-series by co-chromatography with an authentic sample on HPLC equipped with an optical rotation detector. Based on these findings, the structure of cypellogin A was assigned to formula $\mathbf{1}$.

Cypellogin B (2), a light yellow amorphous powder, had the same molecular formula as $\mathbf{1}, \mathrm{C}_{31} \mathrm{H}_{34} \mathrm{O}_{14}$, as revealed by HR-ESI-MS. Its UV spectral pattern $\left(\lambda_{\max } 204,257,356\right.$ $\mathrm{nm}$ ), similar to that of $\mathbf{1}$, suggested it to be an analog of cypellogin A. The ${ }^{1} \mathrm{H}$ - and ${ }^{13} \mathrm{C}$-NMR data of $\mathbf{2}$ assigned by ${ }^{1} \mathrm{H}-{ }^{1} \mathrm{H}$ correlation spectroscopy (COSY), ${ }^{1} \mathrm{H}$-detected heteronuclear multiple quantum coherence spectrum (HMQC) and $\mathrm{HMBC}$, were very similar to $\mathbf{1}$, except for the signal pattern of the sugar protons (Table 1). The sugar residue in $\mathbf{2}$
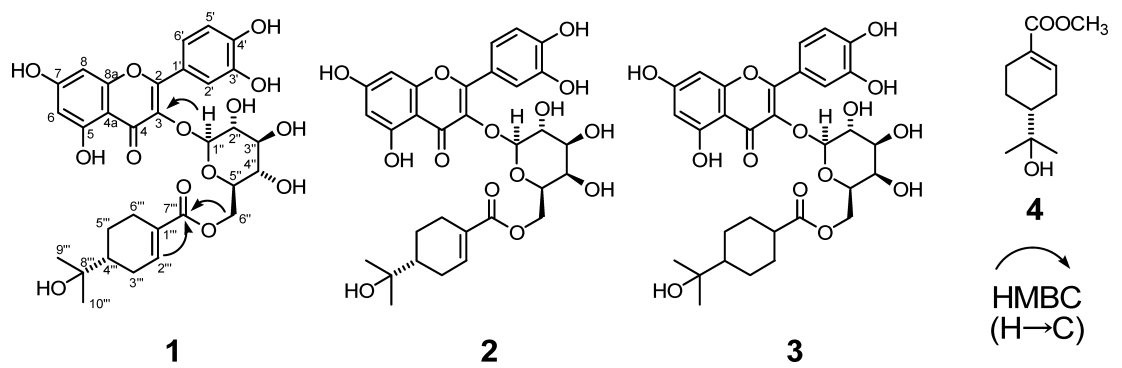

* To whom correspondence should be addressed. e-mail: mikan@shujitsu.ac.jp 
Table 1. ${ }^{1} \mathrm{H}$ - and ${ }^{13} \mathrm{C}-\mathrm{NMR}$ Spectral Data for Cypellogins A (1), B (2) and C (3)

\begin{tabular}{|c|c|c|c|c|c|c|}
\hline \multirow{2}{*}{ Carbon } & \multicolumn{2}{|l|}{1} & \multicolumn{2}{|l|}{2} & \multicolumn{2}{|l|}{3} \\
\hline & $\delta_{\mathrm{H}}$ & $\delta_{\mathrm{C}}$ & $\delta_{\mathrm{H}}$ & $\delta_{\mathrm{C}}$ & $\delta_{\mathrm{H}}$ & $\delta_{\mathrm{C}}$ \\
\hline 2 & & 157.7 & & 157.7 & & 157.8 \\
\hline 3 & & 134.9 & & 135.2 & & 135.4 \\
\hline 4 & & 178.9 & & 178.9 & & 179.0 \\
\hline $4 a$ & & 105.3 & & 105.2 & & 105.4 \\
\hline 5 & & 162.8 & & 162.7 & & 162.8 \\
\hline 6 & $6.25(1 \mathrm{H}, \mathrm{d}, 2)$ & 99.7 & $6.24(1 \mathrm{H}, \mathrm{d}, 2)$ & 99.7 & $6.26(1 \mathrm{H}, \mathrm{d}, 2)$ & 99.7 \\
\hline 7 & & 165.0 & & 165.0 & & 165.2 \\
\hline 8 & $6.48(1 \mathrm{H}, \mathrm{d}, 2)$ & 94.6 & $6.48(1 \mathrm{H}, \mathrm{d}, 2)$ & 94.6 & $6.50(1 \mathrm{H}, \mathrm{d}, 2)$ & 94.6 \\
\hline $8 \mathrm{a}$ & & 158.1 & & 158.0 & & 158.2 \\
\hline $1^{\prime}$ & & 122.6 & & 122.6 & & 122.6 \\
\hline $2^{\prime}$ & $7.74(1 \mathrm{H}, \mathrm{d}, 1.5)$ & 117.1 & $7.87(1 \mathrm{H}, \mathrm{d}, 1.5)$ & 117.3 & $7.87(1 \mathrm{H}, \mathrm{d}, 2.0)$ & 117.4 \\
\hline $3^{\prime}$ & & 145.3 & & 145.2 & & 145.3 \\
\hline $4^{\prime}$ & & 149.1 & & 149.2 & & 149.3 \\
\hline $5^{\prime}$ & $6.90(1 \mathrm{H}, \mathrm{d}, 8.0)$ & 115.7 & $6.90(1 \mathrm{H}, \mathrm{d}, 8.5)$ & 115.7 & $6.92(1 \mathrm{H}, \mathrm{d}, 8.5)$ & 115.7 \\
\hline $6^{\prime}$ & $7.66(1 \mathrm{H}, \mathrm{dd}, 1.5,8.0)$ & 123.1 & $7.69(1 \mathrm{H}, \mathrm{dd}, 1.5,8.5)$ & 122.9 & $7.73(1 \mathrm{H}, \mathrm{dd}, 2.0,8.5)$ & 123.1 \\
\hline $5-\mathrm{OH}$ & $12.47(1 \mathrm{H}, \mathrm{s})$ & & $12.38(1 \mathrm{H}, \mathrm{s})$ & & $12.39(1 \mathrm{H}, \mathrm{s})$ & \\
\hline $1^{\prime \prime}$ & $5.47(1 \mathrm{H}, \mathrm{d}, 7.5)$ & 103.2 & $5.33(1 \mathrm{H}, \mathrm{d}, 7.5)$ & 104.5 & $5.25(1 \mathrm{H}, \mathrm{d}, 8.0)$ & 104.9 \\
\hline $2^{\prime \prime}$ & $3.40-3.60$ & 75.3 & $3.91(1 \mathrm{H}, \mathrm{t}, 7.5)$ & 72.7 & $3.85(1 \mathrm{H}, \mathrm{t}, 8.0)$ & 72.7 \\
\hline $3^{\prime \prime}$ & $3.40-3.60$ & 75.3 & $3.75(1 \mathrm{H}, \mathrm{m})$ & 74.6 & $3.68(1 \mathrm{H}, \mathrm{m})$ & 74.7 \\
\hline $4^{\prime \prime}$ & $3.40-3.60$ & 71.3 & $3.96(1 \mathrm{H}, \mathrm{brs})$ & 69.7 & $3.91(1 \mathrm{H}, \mathrm{m})$ & 69.6 \\
\hline $5^{\prime \prime}$ & $3.40-3.60$ & 77.9 & $3.82(1 \mathrm{H}, \mathrm{t}, 7.5)$ & 74.1 & $3.78(1 \mathrm{H}, \mathrm{m})$ & 74.2 \\
\hline \multirow[t]{2}{*}{$6^{\prime \prime}$} & $4.34(1 \mathrm{H}, \mathrm{dd}, 2.5,11.5)$ & 63.9 & $4.20(2 \mathrm{H}, \mathrm{d}, 6.0)$ & 63.8 & $4.21(1 \mathrm{H}, \mathrm{dd}, 4.5,11.5)$ & 63.9 \\
\hline & $4.08(1 \mathrm{H}, \mathrm{dd}, 7.5,11.5)$ & & & & $4.14(1 \mathrm{H}, \mathrm{dd}, 8.0,11.5)$ & \\
\hline $1^{\prime \prime \prime}$ & & 130.5 & & 130.4 & $2.41(1 \mathrm{H}$, brs $)$ & 39.4 \\
\hline \multirow[t]{2}{*}{$2^{\prime \prime \prime}$} & $6.72(1 \mathrm{H}, \mathrm{m})$ & 140.5 & $6.67(1 \mathrm{H}, \mathrm{m})$ & 140.6 & $1.95(1 \mathrm{H}, \mathrm{m})$ & 28.1 \\
\hline & & & & & $1.26(1 \mathrm{H}, \mathrm{m})$ & \\
\hline \multirow[t]{2}{*}{$3^{\prime \prime \prime}$} & $2.18(1 \mathrm{H}, \mathrm{m})$ & 25.7 & $2.14(1 \mathrm{H}, \mathrm{m})$ & 25.6 & $1.52(1 \mathrm{H}, \mathrm{m})$ & 24.4 \\
\hline & $1.86(1 \mathrm{H}, \mathrm{m})$ & & $1.79-1.86$ & & $1.03(1 \mathrm{H}, \mathrm{m})$ & \\
\hline $4^{\prime \prime \prime}$ & $1.40(1 \mathrm{H}, \mathrm{m})$ & 44.9 & $1.37(1 \mathrm{H}, \mathrm{m})$ & 44.8 & $1.13(1 \mathrm{H}, \mathrm{m})$ & 49.5 \\
\hline \multirow[t]{2}{*}{$5^{\prime \prime \prime}$} & $1.86(1 \mathrm{H}, \mathrm{m})$ & 23.9 & $1.79-1.86$ & 23.8 & $1.52(1 \mathrm{H}, \mathrm{m})$ & 24.4 \\
\hline & $1.06(1 \mathrm{H}, \mathrm{m})$ & & $1.01(1 \mathrm{H}, \mathrm{m})$ & & $1.03(1 \mathrm{H}, \mathrm{m})$ & \\
\hline \multirow[t]{2}{*}{$6^{\prime \prime \prime}$} & $2.26(1 \mathrm{H}, \mathrm{m})$ & 28.0 & $2.24(1 \mathrm{H}, \mathrm{m})$ & 27.9 & $1.95(1 \mathrm{H}, \mathrm{m})$ & 28.1 \\
\hline & $1.86(1 \mathrm{H}, \mathrm{m})$ & & $1.79-1.86$ & & $1.26(1 \mathrm{H}, \mathrm{m})$ & \\
\hline $7^{\prime \prime \prime}$ & & 167.2 & & 167.1 & & 175.0 \\
\hline $8^{\prime \prime \prime}$ & & 72.0 & & 72.1 & & 72.1 \\
\hline $9^{\prime \prime \prime}$ & $1.15(3 \mathrm{H}, \mathrm{s})$ & 27.3 & $1.13(3 \mathrm{H}, \mathrm{s})$ & 27.3 & $0.96(3 \mathrm{H}, \mathrm{s})$ & 27.2 \\
\hline $10^{\prime \prime \prime}$ & $1.14(3 \mathrm{H}, \mathrm{s})$ & 26.8 & $1.12(3 \mathrm{H}, \mathrm{s})$ & 26.7 & $0.96(3 \mathrm{H}, \mathrm{s})$ & 27.0 \\
\hline
\end{tabular}

was determined as $\beta$-D-galactose based on the ${ }^{1} \mathrm{H}$ - and ${ }^{13} \mathrm{C}$ NMR data and acid hydrolysis affording quercetin and $\mathrm{D}-$ galactose, the latter of which was detected by reversed-phase HPLC equipped with an optical rotation detector. The methanolysis of 2 with $\mathrm{NaOMe}$ in $\mathrm{MeOH}$ furnished oleuropeic acid methyl ester $4\left\{[\alpha]_{\mathrm{D}}^{20}+75^{\circ}\left(\mathrm{CHCl}_{3}\right)\right\}$. Based on the above data, the structure of cypellogin $\mathrm{B}$ was determined as formula 2 .

Cypellogin C (3) was obtained as a light yellow amorphous powder. The molecular formula of $\mathbf{3}$ was shown as $\mathrm{C}_{31} \mathrm{H}_{36} \mathrm{O}_{14}$ from HR-ESI-MS giving a pseudomolecular ion peak $\left(\mathrm{m} / z 655.1996[\mathrm{M}+\mathrm{Na}]^{+}\right)$, two mass units larger than that of 2. The ${ }^{1} \mathrm{H}$ - and ${ }^{13} \mathrm{C}-\mathrm{NMR}$ spectra coupled with COSY, HMQC and HMBC data were similar to those of 2. A difference in the ${ }^{13} \mathrm{C}$-NMR spectrum (Table 1) compared with 2 was observed for the presence of additional signals due to a methine (C-1"', $\delta$ 39.4) and a methylene carbon (C-2"', $\delta$ $28.1)$ instead of the two olefinic carbon signals $(\delta 140.6$, 130.4) in $\mathbf{2}$, indicating that a monoterpene unit in $\mathbf{3}$ was a dihydro-derivative of oleuropeic acid. However, the dihydrooleuropeic acid methyl ester was hardly obtainable on the methanolysis of $\mathbf{3}$, probably owing to its high volatility or sublimation. The sugar component was identified as D-galac- tose by acid hydrolysis followed by HPLC analysis. Consequently, the structure of cypellogin $\mathrm{C}$ was elucidated as formula 3, although the cis- or trans-orientation of the 1,4-substituents on the cyclohexane ring remains to be clarified.

\section{Experimental}

General Experimental Procedures UV spectra were measured in $\mathrm{MeOH}$ on a Hitachi U-1800. ESI-MS and HR-ESI-MS were recorded on a Bruker Micro TOF LC (solvent: $\mathrm{MeOH}$; flow rate $200 \mu \mathrm{l} / \mathrm{min}$ ). ${ }^{1} \mathrm{H}$ - and ${ }^{13} \mathrm{C}$ NMR spectra were recorded on JEOL NM-ECA-500 $\left(500 \mathrm{MHz}\right.$ for ${ }^{1} \mathrm{H}$, $126 \mathrm{MHz}$ for ${ }^{13} \mathrm{C}$ ) and chemical shifts are given as $\delta(\mathrm{ppm})$ value referenced to the signals of acetone- $d_{6}\left(\delta_{\mathrm{H}} 2.04, \delta_{\mathrm{C}} 29.8\right)$. Optical rotations were measured with a JASCO P-1020 polarimeter. Diaion HP-20 (Mitsubishi Kasei Co.), Toyopearl HW-40 (fine grade) (Tosoh Corp.) and YMC-GEL ODS AQ 120-50S (YMC Co., Ltd.) were used for column chromatography. Preparative HPLC was performed using an ODS column (UG-80, $20 \mathrm{~mm}$ i.d. $\times 250 \mathrm{~mm}$, Shiseido Co., Japan) and developed isocratically at room temperature with $10 \mathrm{~mm} \mathrm{H} \mathrm{H}_{3} \mathrm{PO}_{4} / 10 \mathrm{mM} \mathrm{KH}_{2} \mathrm{PO}_{4} / \mathrm{MeCN}$ (solvent A; 40/40/20) and $\mathrm{H}_{2} \mathrm{O} / \mathrm{AcOH} / \mathrm{MeCN}$ (solvent $\mathrm{B} ; 75 / 5 / 20$ ). The sugar fraction was analyzed by reversed-phase HPLC with an optical rotation detector (JASCO OR-2090) [column, YMC-Pack polyamine II $(4.6 \mathrm{~mm}$ i.d. $\times 150 \mathrm{~mm})$; solvent, $\mathrm{MeCN} / \mathrm{H}_{2} \mathrm{O}(75 / 25)$; column temperature, $\left.40^{\circ} \mathrm{C}\right]$. Analytical and preparative TLC were carried out on precoated silica gel plates (Merck, $0.25 \mathrm{~mm}$ thickness).

Plant Materials Leaves of E. cypellocarpa were collected at Awaji Farm Park in August 2003. The specimen was deposited in the Herbarium, Faculty of Pharmaceutical Sciences, Okayama University. 
Extraction and Isolation The air-dried leaves $(3.0 \mathrm{~kg})$ were powdered and extracted with $\mathrm{MeOH}(121 \times 3)$. The $\mathrm{MeOH}$ extract $(863.0 \mathrm{~g})$ was suspended in $\mathrm{H}_{2} \mathrm{O}$ (11) and extracted successively with $n$-hexane $(11 \times 6)$, AcOEt $(11 \times 6)$ and $n$-BuOH $(11 \times 6)$ to yield an $n$-hexane extract $(187.0 \mathrm{~g})$, AcOEt extract $(250.2 \mathrm{~g}), n-\mathrm{BuOH}$ extract $(166.7 \mathrm{~g})$ and $\mathrm{H}_{2} \mathrm{O}$ extract $(249.2 \mathrm{~g})$. A part of the AcOEt extract $(200 \mathrm{~g})$ was chromatographed on Diaion $\mathrm{HP}-20$ with $\mathrm{MeOH} / \mathrm{H}_{2} \mathrm{O}(10 / 90,30 / 70,50 / 50,70 / 30,100 / 0)$ system to give five fractions (Frs. $1-5$ ). The Fr. 3 and 4 were further fractionated separately by column chromatography on Toyopearl $\mathrm{HW}-40$ with $\mathrm{MeOH} / \mathrm{H}_{2} \mathrm{O}$ $(10 / 90,30 / 70,50 / 50,70 / 30,100 / 0)$ system and then acetone/ $\mathrm{MeOH} / \mathrm{H}_{2} \mathrm{O}$ $(10: 70: 20,70: 0: 30)$ to give fractions $3-1-3-18$ and $4-1-4-17$, respectively.

Fractions 3-2, 3-9 and 3-12 were purified by preparative HPLC (solvent A) to yield icariside $\mathrm{H}_{1}(113 \mathrm{mg})$ from the fraction $3-2$, benzyl $6^{\prime}-O$-galloyl$\beta$-glucopyranoside ( $25 \mathrm{mg})$ from the fraction $3-9$, quercetin $3-O-\left(6^{\prime \prime}-O\right.$-galloyl)- $\beta$-D-galactopyranoside ( $18 \mathrm{mg}$ ) from the fraction $3-12$.

Fraction 4-3 was chromatographed over YMC-GEL ODS-A 120-S50 with aqueous $\mathrm{MeOH}$ in a stepwise gradient. The $40 \% \mathrm{MeOH}$ eluate gave 3-Omethylellagic acid $4^{\prime}-O$-rhamnoside $(6 \mathrm{mg})$. The $50 \% \mathrm{MeOH}$ eluate was further purified by preparative HPLC (solvent B) to yield cypellogin A (1, $73 \mathrm{mg})$, cypellogin B $(2,137 \mathrm{mg})$, cypellogin $\mathrm{C}(\mathbf{3}, 31 \mathrm{mg})$, resinoside A $(15 \mathrm{mg})$ and resinoside B $(14 \mathrm{mg})$. Fraction 4-5 was separated by YMC-GEL ODS-A 120-S50 column chromatography with aqueous $\mathrm{MeOH}$ in a stepwise gradient. Guaijaverin $(86 \mathrm{mg})$ and quercitrin $(39 \mathrm{mg})$ were obtained by HPLC purification (solvent A) of the $50 \% \mathrm{MeOH}$ eluate.

Cypellogin A (1): Light yellow amorphous powder, $[\alpha]_{\mathrm{D}}-7^{\circ}(c=0.1$, $\mathrm{MeOH})$. UV $\lambda_{\max } \mathrm{MeOH} \mathrm{nm}(\log \varepsilon): 204$ (4.71), 257 (4.30), 356 (4.22). ESI-MS $m / z 653[\mathrm{M}+\mathrm{Na}]^{+}$. HR-ESI-MS $m / z$ 653.1852 [M+Na] ${ }^{+}(\mathrm{Calcd}$ for $\left.\mathrm{C}_{31} \mathrm{H}_{34} \mathrm{O}_{14}+\mathrm{Na}, 653.1841\right)$. ${ }^{1} \mathrm{H}-,{ }^{13} \mathrm{C}$-NMR spectral data, see Table 1 .

Cypellogin $\mathrm{B}(2)$ : Light yellow amorphous powder, $[\alpha]_{\mathrm{D}}+47^{\circ}(c=0.1$, $\mathrm{MeOH})$. UV $\lambda_{\max } \mathrm{MeOH} \mathrm{nm}(\log \varepsilon): 204$ (4.71), 257 (4.30), 356 (4.22). ESI-MS $m / z 653[\mathrm{M}+\mathrm{Na}]^{+}$. HR-ESI-MS $m / z$ 653.1852 [M+Na] ${ }^{+}(\mathrm{Calcd}$ for $\left.\mathrm{C}_{31} \mathrm{H}_{34} \mathrm{O}_{14}+\mathrm{Na}, 653.1841\right) .{ }^{1} \mathrm{H}-,{ }^{13} \mathrm{C}-\mathrm{NMR}$ spectral data, see Table 1 .

Cypellogin $\mathrm{C}(3)$ : Light yellow amorphous powder, $[\alpha]_{\mathrm{D}}+10^{\circ}(c=0.1$, $\mathrm{MeOH})$. UV $\lambda_{\max } \mathrm{MeOH} \mathrm{nm}(\log \varepsilon): 205$ (4.71), 257 (4.43), 360 (4.35). ESI-MS: $m / z 655$ [M+Na $^{+}$, HR-ESI-MS m/z $655.1996[\mathrm{M}+\mathrm{Na}]^{+}(\mathrm{Calcd}$ for $\left.\mathrm{C}_{31} \mathrm{H}_{36} \mathrm{O}_{14}+\mathrm{Na}, 655.1997\right) .{ }^{1} \mathrm{H}-,{ }^{13} \mathrm{C}-\mathrm{NMR}$ spectral data, see Table 1.

Acid Hydrolysis of 1,2 and 3 A solution of $1(1.0 \mathrm{mg})$ in $1 \mathrm{M} \mathrm{HCl}$ $(1.0 \mathrm{ml})$ was heated for $1 \mathrm{~h}$ in a boiling water bath. After cooling, the reaction mixture was applied to a Mega Bond Elut $\mathrm{C}_{18}$ cartridge column, and eluted first with $\mathrm{H}_{2} \mathrm{O}$ and then with $\mathrm{MeOH}$. The $\mathrm{H}_{2} \mathrm{O}$ fraction was passed through a short Amberlite IRA-400 column and evaporated to give D-glucose, which was identified based on the HPLC retention time and optical rotation. The $\mathrm{MeOH}$ fraction was evaporated to yield quercetin. Acid hydrolysis of $\mathbf{2}$ and $\mathbf{3}$ was similarly carried out to give D-galactose as well as quercetin.

Methanolysis of 1 and 2 A solution of $1(6 \mathrm{mg})$ in $1 \% \mathrm{NaOMe} / \mathrm{MeOH}$ $(1.0 \mathrm{ml})$ was kept standing at room temperature for $11 \mathrm{~h}$. After the addition of two drops of $\mathrm{AcOH}$, the concentrated solution was subjected to column chromatography over silica gel followed by preparative TLC (toluene-acetone, $4: 1)$ to yield $(+)$-oleuropeic acid methyl ester $(4),[\alpha]_{\mathrm{D}}+85^{\circ}$ $\left(c=0.02, \mathrm{CHCl}_{3}\right)$. Cypellogin $\mathrm{B}(2)(5 \mathrm{mg})$ was similarly methanolyzed to give $4,[\alpha]_{\mathrm{D}}+75^{\circ}\left(c=0.02, \mathrm{CHCl}_{3}\right)$. Compound 4 obtained from both $\mathbf{1}$ and 2 was identified using an authentic specimen derived from cypellocarpin $\mathrm{A}^{3}{ }^{3)}$

Acknowledgements The authors are grateful to Awaji Farm Park for the generous supply of the plant materials.

\section{References}

1) Ghisalberti E. L., Phytochemistry, 41, 7-22 (1996).

2) Hegnauer R., "Chemotaxonomie der Pflanzen," Vol. IX, Birkhauser, Basel, 1986.

3) Ito H., Koreishi M., Tokuda H., Nishino H., Yoshida T., J. Nat. Prod., 63, 1253-1257 (2000)

4) Matsushita H., Miyase T., Ueno A., Phytochemistry, 30, 2025-2027 (1991).

5) Isaza J. H., Ito H., Yoshida T., Phytochemistry, 58, 321-327 (2001).

6) Kim J. P., Lee I. K., Yun B. S., Chung S. H., Shim G. S., Koshino H., Yoo I. D., Phytochemistry, 57, 587-591 (2001).

7) Hyodo S., Etoh H., Yamashita N., Sakata K., Ina K., Biosci. Biotech. Biochem., 56, 138 (1992).

8) Manns D., Hartmann R., Planta Medica, 60, 467-469 (1994). 